\title{
BMJ Open Improving primary care for diabetes and hypertension: findings from implementation research in rural South India
}

Dorothy Lall (D , ${ }^{1}$ Nora Engel, ${ }^{2}$ Prashanth N Srinivasan, ${ }^{3}$ Narayanan Devadasan, ${ }^{4}$ Klasien Horstman, ${ }^{2}$ Bart Criel $^{5}$

To cite: Lall D, Engel N, Srinivasan PN, et al. Improving primary care for diabetes and hypertension: findings from implementation research in rural South India. BMJ Open 2020;10:e040271. doi:10.1136/ bmjopen-2020-040271

- Prepublication history and additional material for this paper are available online. To view these files, please visit the journal online (http://dx.doi. org/10.1136/bmjopen-2020040271).

Received 08 May 2020 Revised 30 September 2020 Accepted 14 November 2020

Check for updates

(C) Author(s) (or their employer(s)) 2020. Re-use permitted under CC BY-NC. No commercial re-use. See rights and permissions. Published by BMJ.

For numbered affiliations see end of article.

Correspondence to

Dr Dorothy Lall;

dorothylall@gmail.com

\section{ABSTRACT}

Background Chronic conditions are a leading cause of death and disability worldwide. Low-income and middle-income countries such as India bear a significant proportion of this global burden. Redesigning primary care from an acute-care model to a model that facilitates chronic care is a challenge and requires interventions at multiple levels.

Objectives In this intervention study, we aimed to strengthen primary care for diabetes and hypertension at publicly funded primary healthcare centres (PHCs) in rural South India.

Design and methods The complexities of transforming the delivery of primary care motivated us to use a 'theory of change' approach to design, implement and evaluate the interventions. We used both quantitative and qualitative data collection methods. Data from patient records regarding processes of care, glycaemic and blood pressure control, interviews with patients, observations and field notes were used to analyse what changes occurred and why.

Interventions We implemented the interventions for 9 months at three PHCs: (1) rationalise workflow to include essential tasks like counselling and measurement of blood pressure/blood glucose at each visit; (2) distribute clinical tasks among staff; (3) retain clinical records at the health facility and (4) capacity building of staff.

Results We found that interventions were implemented at all three PHCs for the first 4 months but did not continue at two of the PHCs. This fadeout was most likely the result of staff transfers and a doctor's reluctance to share tasks. The availability of an additional staff member in the role of a coordinator most likely influenced the relative success of implementation at one PHC.

Conclusion These findings draw attention to the need for building teams in primary care for managing chronic conditions. The role of a coordinator emerged as an important consideration, as did the need for a stable core of staff to provide continuity of care.

\section{BACKGROUND}

Non-communicable diseases (NCDs) are the leading causes of death and disability worldwide. ${ }^{1}$ In 2016, based on the prevalence rates for NCDs in India, one in every five persons
Strengths and limitations of this study

- This study reports implementation in the 'real-world setting' that tests local solutions, co-designed with implementers to improve primary care for diabetes and hypertension in India.

- We use a theory of change approach in the design and evaluation of the interventions in an attempt to understand the effect and why change did or did not occur.

- The study was limited by the short duration of implementation (9 months) and we acknowledge that this may not have been adequate to produce the changes we have evaluated.

- The study was conducted at three public primary care faciitlies in rural South India with contextual differences and thus findings are not generalisable.

- A major limitation of the study was the lack of involvement of patients in planning and design due to local cultural influences.

had a chronic NCD. ${ }^{2}$ Caring for persons with a chronic condition is a struggle for most health systems worldwide because traditionally they are designed to deliver care for acute diseases. ${ }^{3}$ The care for persons with a chronic condition requires an engagement with the healthcare delivery system over extended periods, coordinated inputs from a wide range of health professionals, access to essential medicines and monitoring systems that are optimally embedded within a system that promotes patient empowerment. ${ }^{3}$ It has been argued that the primary level of healthcare is best suited to the care of persons with a chronic condition. ${ }^{45}$ A primary level of care close to people's homes ensures continuity and coordination, has many positive effects on clinical outcomes, quality of care and is cost-effective. ${ }^{6} 7$

Chronic care at the primary level is challenging in low-income and middle-income countries such as India. ${ }^{89}$ Primary care often 
lacks essential resources such as trained professionals, access to medicines and robust information systems, in part because of chronic underfunding of healthcare. ${ }^{10}$ ${ }^{-11}$ In addition, outpatient care at the primary level in India is oriented towards acute conditions. Clinical records, for example, are not retained at public health facilities because they are not considered essential for care of acute episodic diseases. Similarly, counselling for lifestyle modification has not historically been viewed as necessary. As a result, despite the escalating burden of chronic conditions, primary care in India is mostly unprepared to deliver care for a person with chronic conditions. ${ }^{12} 13$

Thus, a redesign of primary care is needed. Models such as the chronic care model, by Wagner $e t$ al, and the innovative chronic care model of WHO provide guidance for the redesign of primary healthcare facilities. ${ }^{14}$ These models suggest that developing clinical information systems, decision support and delivery designs that enable self-management positively affect outcomes of care. ${ }^{15}$ Most models highlight the role of proactive teams at the primary care facilities that deliver care while linking to community resources. ${ }^{14}$ Roles of the staff at primary care facilities and the community health worker would need to be redefined for chronic care. However, changing care delivery at primary care facilities is difficult. A large number of multilevel contextual influences related to the policy environment, organisational context, health professionals in primary care and intervention characteristics affect the ability of primary care delivery systems and its actors to change. ${ }^{16}$ This complexity renders traditional approaches to implementation and evaluation insufficient. ${ }^{17}$

In this study, we aimed to improve the delivery of care for chronic NCDs at primary healthcare centres (PHCs) in rural South India. We use a theory of change (ToC) approach to guide the implementation and evaluation of interventions. This approach is increasingly used to understand how change occurs in complex healthcare settings. ${ }^{17}$

The interventions in this study were co-designed with implementers and addressed critical gaps in service delivery for chronic care at the primary care level in India that have been previously reported. ${ }^{18}$ The team expected the interventions to result in continuous care, appropriate treatments, counselling support and ultimately good control of blood pressure or blood glucose levels for patients enrolled at the primary care facility.

In this paper, we present an evaluation of these interventions, identifying what changed and the effect of these changes on the anticipated outcomes. We also offer plausible explanations for how change occurred and why all of the intended changes were not achieved. We have reported an analysis of the local contextual influences on implementation and refer to it in this paper to communicate a complete understanding of why changes did or did not occur. ${ }^{19}$ The insights we gained have implications for the redesign of primary care for chronic conditions and may be informative for similarly resource-constrained settings.

\section{METHODS}

We chose a case study approach and used both quantitative and qualitative methods for data collection ${ }^{20}$ to assess the interventions. This approach was best suited to the objectives of determining which changes occurred and their effect on clinical outcomes and understanding how and why these changes occurred. A package of interventions to improve service delivery for diabetes and hypertension was implemented at three publicly funded primary PHCs in the Kolar district of Karnataka state in South India. We chose to study three cases rather than only one to allow comparisons across the similar settings. Interventions were implemented for 9 months, from March to December 2018, preceded by 3 months of preparation to fine tune the interventions and develop a ToC with the healthcare team at the PHCs.

\section{Setting}

Healthcare delivery in the public sector in India is organised into three tiers: primary, secondary and tertiary levels of care. The infrastructure for the delivery of primary care health services includes PHCs for up to 30000 people, and subcentres for up to 5000 people. A PHC is the first point of contact with a medical doctor and is intended to provide integrated curative and preventive healthcare with an emphasis on disease prevention and promotion of health. ${ }^{21}$ The team at a PHC includes a medical doctor, nurses, a lab technician and a pharmacist. At the subcentre, an auxiliary nurse midwife and community health workers known as accredited social health activists (ASHAs) are the link between the community and medical services. ASHA is usually from the community and visits homes to provide health advice and assistance. The focus of work is maternal and child health, which is recompensed with a performance-based honorarium.

The outpatient services at a PHC run for 6 hours per day ( 4 hours in the morning and 2 hours in the afternoon) for 6 days a week. Minimum outpatient attendance is expected to be 40 patients per day, although the range is 20-150 in the PHCs of Karnataka State. An outpatient clinic typically offers a mix of antenatal care, care for common communicable diseases and increasingly, care for NCDs such as diabetes. Specific disease-control activities are delivered at the PHC as specified in the relevant national disease control programmes. There is a national programme for the prevention and control of diabetes and hypertension that defines a package of activities for each level of the healthcare delivery system including a PHC.

Kolar district is one of the 30 districts in the state of Karnataka and was selected because of its proximity to Bengaluru city where the Institute of Public Health is located. The district is fairly representative of districts within the state with respect to disease burden and public 


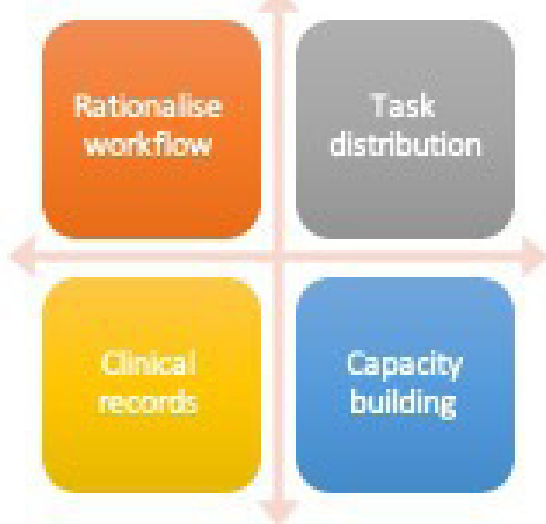

Figure 1 Intervention package.

healthcare infrastructure. In addition, Kolar was one of the five districts that was selected for pilot implementation of the national programme for NCD care when it was launched in 2009 .

\section{Selection of participating PHCs: intervention case studies}

Three PHCs were selected, in consultation with the district health officer and based on the following criteria: (1) providing care for diabetes and hypertension; (2) availability of health professionals at the facility who would remain in service for at least 1 year, as assessed by the district health officer and (3) willingness of the doctors and teams to participate in the study. The PHCs had similar basic infrastructure and staff, according to the national guideline for $\mathrm{PHCs},{ }^{22}$ which included a medical doctor, two nurses, a laboratory technician and a pharmacist. One of the three PHCs had an additional staff member recruited under a pilot project to enrol citizens in a national healthcare database. This staff member had no prior experience in healthcare delivery but was familiar with information technology and volunteered to participate in the implementation of the interventions in addition to the activities of the project.

\section{Design of the intervention package}

We started with a gap analysis to identify aspects of service delivery for chronic NCDs that needed strengthening. The findings of this analysis have been reported previously. ${ }^{18}$
The gaps identified were as follows: a lack of clinical information retained at the facilities, impeding continuity of care; lack of counselling to support patients for selfmanagement and a fragmentation of services delivery. ${ }^{9}$ A list of interventions to address the gaps was prepared, taking into account recommendations of national and international guidelines on NCD care, such as the WHO Package of Essential services for NCDs. ${ }^{23} 24$ The guidelines identify risk assessment, measurement of blood pressure and blood glucose, foot examination, counselling, prescribing medication, recording clinical details and dispensing medication as essential tasks. Risk assessment, counselling, foot examination and recording of clinical information were not a routine practice at the selected PHCs or at most PHCs in India.

We held an average of four consultations at each of the PHCs and shared the list of essential tasks as well as the gaps in service delivery with the team. The staff discussed changes required in the current delivery of services to operationalise the list of essential tasks. In the first round of discussions, we arrived at a common understanding of the risk factors for diabetes and hypertension. Subsequent discussions focused on caring for persons with these conditions, the feasibility of implementing these tasks to list possible interventions. Finally, after a prioritisation exercise, the agreed-upon intervention package which was implemented at the three sites included: (1) rationalise and reorganise the workflow to include essential tasks such as counselling and measurement of blood pressure/blood glucose at each visit; (2) distribute and share clinical tasks among the team at the facility; (3) initiate a clinical record for patients with diabetes or hypertension to be retained at the healthcare facility and retrieved at each visit for follow-up after enrolment and (4) capacity building of staff (figure 1).

The workflow prior to the interventions is depicted in figure 2 and included patients visiting the doctor, who would instruct the lab technician or nurse to measure blood glucose or blood pressure. Patients would then return to the doctor, who counselled them and prescribed medication that was dispensed by the pharmacist. Patients would consult the doctor again to confirm the dose and timing of medication. On average, each patient made

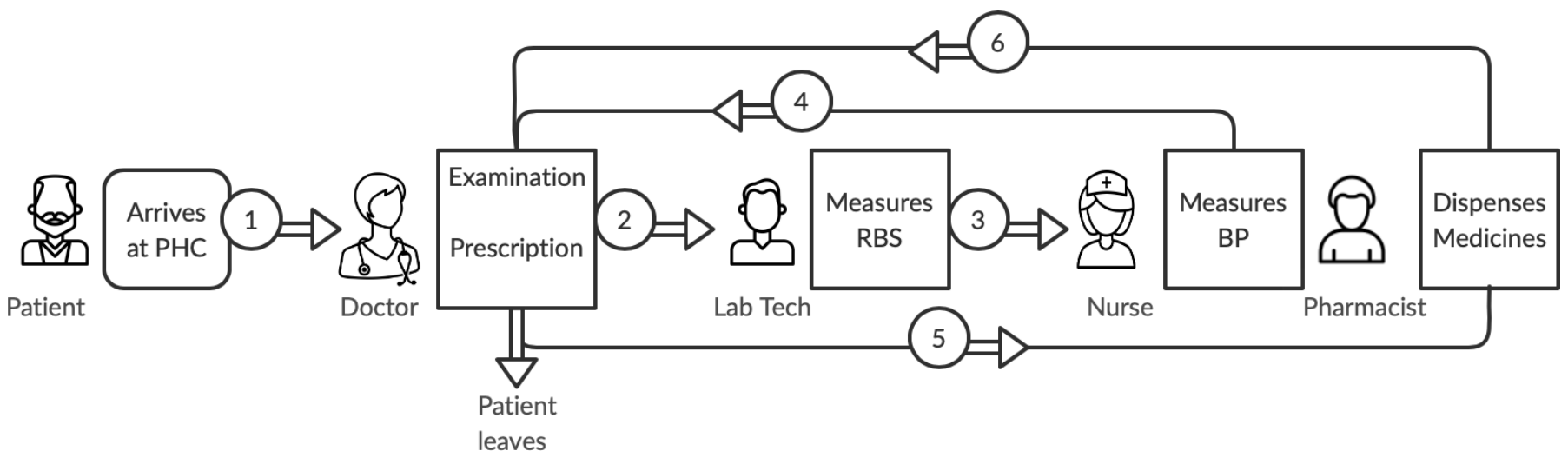

Figure 2 Workflow prior to implementation. BP, blood pressure; PHC, primary healthcare centre; RBS, random blood sugar. 
Patient
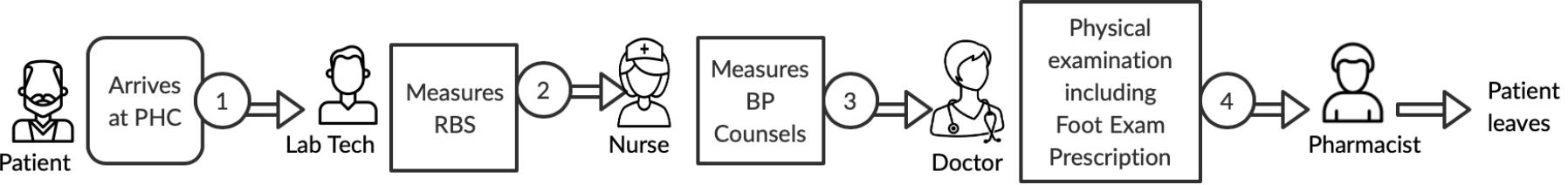

Figure 3 Changed workflow. BP, blood pressure; PHC, primary healthcare centre; RBS, random blood sugar.

three trips to the doctor at each visit to the facility, each time having to wait in a queue outside the exam room (figure 2). The workflow was rationalised and streamlined to enable patients to make only one trip to the doctor during a single visit at the PHC (figure 3).

A recording format was co-designed with the staff to record essential information such as risk factors, measurement of blood pressure and fasting or random blood glucose at each visit and the medication prescribed (online supplemental Annex 1). The recording format was piloted and refined with inputs from the staff that would eventually use the tool. The staff recorded the tasks that they completed in this recording format, for example, the laboratory technician would record the blood glucose, and the nurse would record risk factors identified and counselling given.

The staff expressed having a lack of the needed knowledge and skills for some of the tasks, such as counselling and foot examination. Therefore, training and orientation sessions were conducted before implementation of the interventions to build up this capacity. Standard treatment guidelines recommended by the government of India for diabetes and hypertension were discussed with the medical officer. ${ }^{25}$ Each of the capacity-building sessions was interactive, case-based and practical and facilitated by the primary author and a research associate using publicly available resources prescribed in the national programme for NCD control.

\section{Patient and public involvement}

Patients were not directly involved in the design of the interventions due to local contextual difficulties. However, feedback from patients was sought at regular intervals to fine tune the interventions. Patients also participated in the final evaluation at PHC 2. We invited patients for the final dissemination meeting with staff at the PHC.

\section{Theory of change}

The ToC approach emerged from the work of the Aspen Institute Roundtable on Community Change in the $1990 \mathrm{~s}^{26}$ and has origins in theory-driven approaches for evaluation. The basic tenet is that understanding the theory underlying a programme is necessary to understand whether and how the interventions work. ${ }^{27}$

The core elements of any ToC are (1) interventions to bring about outcomes; (2) affect pathways of change that illustrate the relationship among interventions, outcomes and impact; (3) indicators that are defined with enough specificity to measure the outcomes and (4) assumptions that underlie the theory. ${ }^{28}$ The ToC is in fact a map created to provide an overarching theoretical framework that identifies knowledge gaps, enabling the choice of the most appropriate evaluation methods. It integrates process and effectiveness evaluation under a single theoretical framework. ${ }^{27}$

We developed the ToC in consultation with the implementers and district health officers to arrive at a shared understanding of the interventions. We also referred to the literature on implementation research on NCD management to refine the assumptions underlying the ToC. ${ }^{29} 30$

The expected outcomes at the end of a year were as follows: (1) adequate control of fasting blood glucose levels and blood pressure for patients with diabetes and hypertension and (2) regular follow-up ( $>3$ visits in the year) for patients enrolled in care. Control of blood glucose and blood pressure would be expected to lead ultimately to lower complication rates and contribute to better quality of life for persons with diabetes or hypertension (figure 4). The impact of the intervention package would be expected to accumulate over a prolonged period, but for the purposes of this study, we limited ourselves to the assessment of outcomes and not the impact. The extent of evaluation is referred to as the 'ceiling of accountability' in ToC literature. ${ }^{27}$

Expected steps in the pathways of change were patient acceptance of the intervention, adherence to medication and lifestyle modification, along with guideline-based and clinical information-based treatments and reminders for follow-up visits.

The assumptions underlying the ToC were that (1) the training sessions would increase staff competence in fulfilling essential tasks and enable application of skills such as counselling and guideline-based treatments; (2) patients would adhere to treatment regimens and lifestyle advice and (3) patients would heed the reminder to follow-up at the PHC (table 1).

\section{Outcomes and assessments}

The ToC guided and defined the assessments. The outcomes we measured were (1) the number of patients who came for follow-up visits, (2) average fasting blood glucose measurements in person-months of follow-up and (3) average blood pressure measurements among patients who attended a follow-up visit.

Process indicators identified as being coherent with the ToC were: (1) number of patients with a completed risk assessment, (2) number of patients who had blood pressure measured, (3) number of patients who had fasting blood glucose measured, (4) number of patients who had a foot examination done, (5) number of patients who had 
Table 1 Interventions, rationale and assumptions in the theory of change

\begin{tabular}{|c|c|c|}
\hline Intervention & Rationale & Assumptions \\
\hline 1. Change in workflow & $\begin{array}{l}\text { Identification of essential tasks in the workflow would enable staff to } \\
\text { perform tasks. }\end{array}$ & \multirow{5}{*}{$\begin{array}{l}\text { 1. Skills developed } \\
\text { during training } \\
\text { sessions will be } \\
\text { applied, especially } \\
\text { treatment decision- } \\
\text { making and } \\
\text { counselling skills. } \\
\text { 2. Patients will adhere } \\
\text { to treatment regimens. } \\
\text { 3. Patients will heed } \\
\text { the reminder to follow- } \\
\text { up at PHC. }\end{array}$} \\
\hline 2. Tasks distribution among staff & $\begin{array}{l}\text { Shared, distributed tasks ensure team-based care and decrease } \\
\text { load on individual members. }\end{array}$ & \\
\hline 3. Training & Enables staff to perform the assigned tasks. & \\
\hline $\begin{array}{l}\text { 5. Counselling for adherence } \\
\text { to medication and lifestyle } \\
\text { modification }\end{array}$ & $\begin{array}{l}\text { Evidence from other studies support that counselling enables } \\
\text { patients to make and sustain healthy lifestyle choices. }\end{array}$ & \\
\hline $\begin{array}{l}\text { 6. ASHA to remind patients in } \\
\text { their homes }\end{array}$ & $\begin{array}{l}\text { ASHAs are the link between healthcare services and the community } \\
\text { and are best placed to support patients at their homes. }\end{array}$ & \\
\hline
\end{tabular}

ASHA, accredited social health activist.

counselling at each of the three PHCs and (6) number of patients whom ASHAs visited at home.

\section{Data collection procedures}

Data for measurement of the included indicators were extracted from the facility-retained patient records each month and entered into an Excel spreadsheet by a research associate. Data entry checks were included in the spreadsheet to ensure data quality, and the primary author verified $10 \%$ of the entries. The research associate and the primary author made an average of 14 visits to each of the PHCs during the implementation phase. During these visits, field notes and observations were recorded. Observations were specifically guided by the ToC and contradiction or agreement with the pathways of change and underlying assumptions were looked for and noted.
We conducted semi-structured interviews at the end of the intervention period with selected patients using an interview guide (online supplemental Annex 2) to assess patient perception of change and acceptance of the interventions. Patients who came for regular follow-up visits ( $>3$ visits) at PHC 2 were selected because this implementation was sustained throughout the 9 months at this PHC only. In total, we conducted nine interviews (see patient characteristics in table 2). Interviews were conducted with due attention to privacy at the PHC, in the local language after informed consent was obtained, and lasted for 30 min on average. The research associate translated and transcribed the interviews, and the primary author verified them. At each visit to the PHC, the research team took field notes, guided by the ToC.

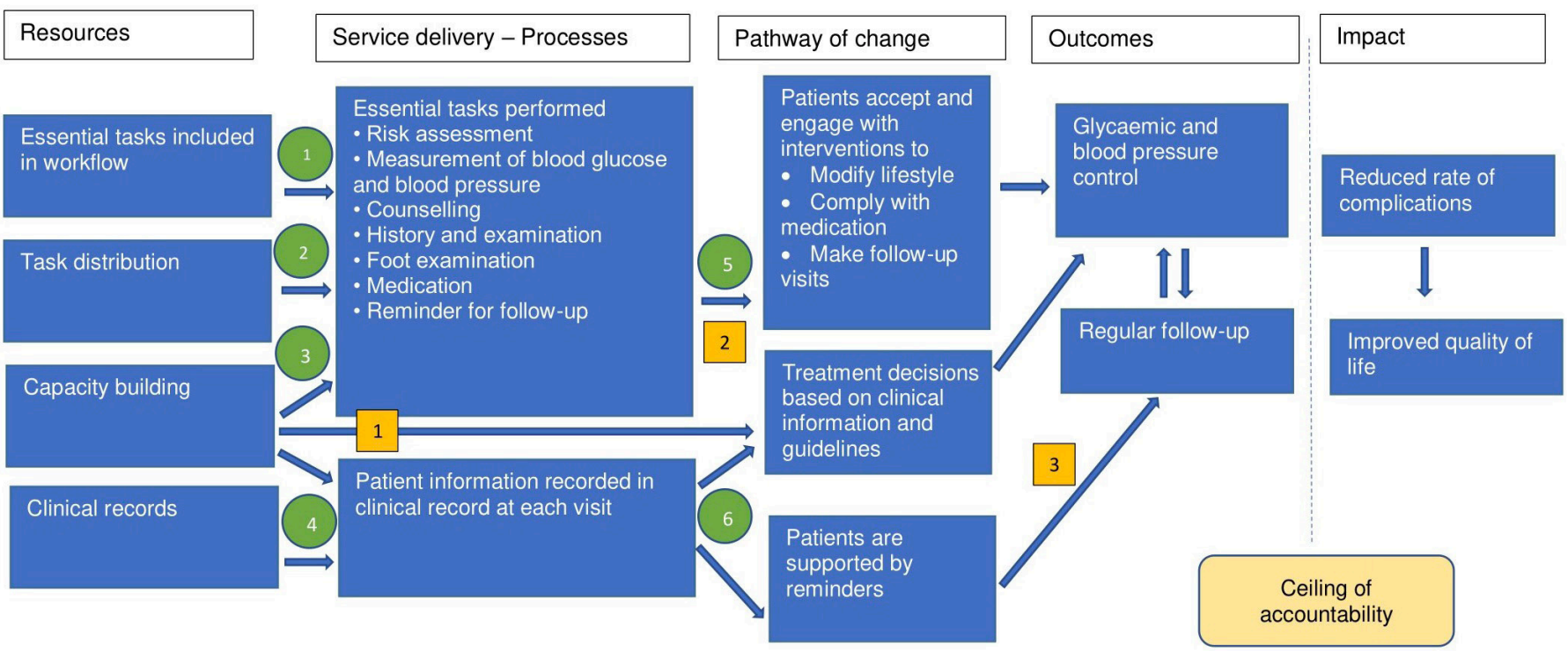

Figure 4 Theory of change. Text in blue boxes represent inputs, green circles depict interventions and yellow boxes point to assumptions made. Please refer to table 1 for more details. 


\section{Data analysis}

Quantitative data were entered into the spreadsheet and analysed for proportions and incidence of outcomes by person-months in care, using IBM SPSS Statistics for Windows, V.20.0 (IBM, Armonk, New York, USA). The average fasting blood glucose or blood pressure levels and the number of visits per person-months of follow-up were determined. Process indicators were analysed as percentages and proportions for each PHC separately. Trends over the 9 months of implementation (March-December 2018) were analysed.

The qualitative data (field notes, interview transcripts and observations) were processed using NVivo (QSR International, V.11, 2015) and analysed both deductively and inductively. Codes were developed a priori from the ToC to include processes of care such as health records, counselling, evaluation of care, medicines and staff roles. Codes were also identified from the data that did not fit the a priori codes. These were then broadly categorised for a clearer understanding of what changed, what did not change and why change did or did not occur. Data from field notes, observations and interviews were triangulated with the quantitative data from the clinical records to yield possible explanations for the observed findings and explore pathways of change. There was no discordance among data from different sources.

\section{RESULTS}

We first present the number of patients enrolled at each PHC, their characteristics and the proportion that came for follow-up visits. We then describe what changed at each PHC and how long these changes continued. We also identify what we intended to change but could not and then offer some possible explanations for why these changes did or did not occur, based on the data. For data from PHC 2, we relate the outcomes of fasting blood glucose and blood pressure control with personmonths of follow-up by patients over the 9 months of the

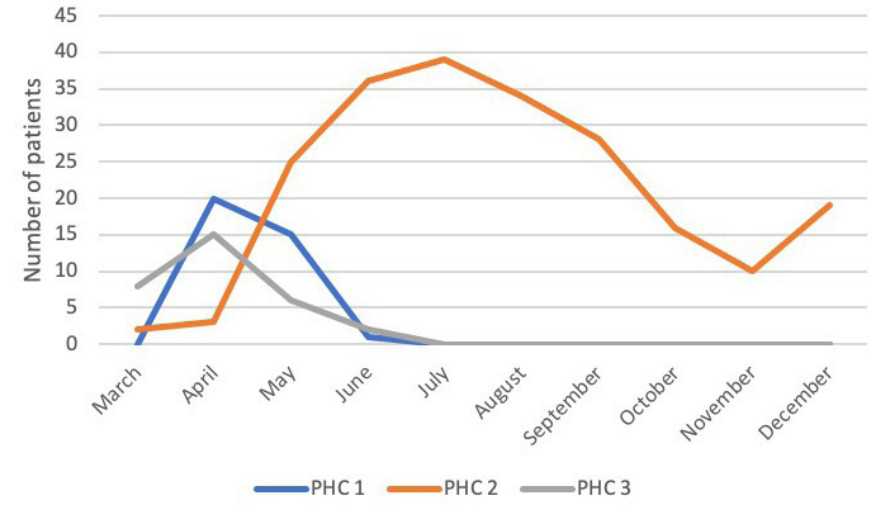

Figure 5 Total number of new patients visiting the primary healthcare centre $(\mathrm{PHC})$ and enrolling for care monthwise during the intervention period at the three PHCs.

implementation. The interventions did not continue at two PHCs, so we were unable to study outcomes at PHC 1 and PHC 3.

The total numbers of NCD patients enrolled at PHCs 1, 2 and 3 were 36, 212 and 31, respectively (figure 5). The average age of the patients enrolled at the PHCs was 59.3 (SD 10.1), 61.5 (SD 10.9) and 64.5 (SD 9.1) at PHC 1, 2 and 3, respectively. PHC 1 and PHC 3 did not enrol any new patients after the first few months.

Patients who visited the PHCs were usually asked to return for a follow-up visit within 1-3 months. Medicines were dispensed accordingly, and the follow-up date was noted in the patient clinical record. This date was also recorded in a patient-retained notebook that was a regular practice at the PHCs (not an intervention of this study). Only $33 \%, 42 \%$ and $58 \%$ of patients who were enrolled for care at PHCs 1, 2 and 3, respectively, returned for the first follow-up visit. Of the patients who came for the second follow-up visit, $66 \%, 50 \%$ and $39 \%$ returned for a third follow-up visit (figure 6).

The proportion of women using PHCs for diabetes or hypertension care was $60 \%, 62.7 \%$ and $58 \%$ at PHCs 1,2

Table 2 Characteristics of participants for in-depth interviews

\begin{tabular}{lllllll}
\hline S no. & Sex & Age (years) & $\begin{array}{l}\text { Distance from } \\
\text { the PHC }(\mathbf{k m})\end{array}$ & $\begin{array}{l}\text { Duration of } \\
\text { condition } \\
\text { (years) }\end{array}$ & $\begin{array}{l}\text { Duration of seeking } \\
\text { care at PHC (years) }\end{array}$ & DM/HTN/Both \\
\hline 1 & $\mathrm{~F}$ & $60-70$ & $<3$ & 1.5 & $1-2$ & HTN \\
\hline 2 & $\mathrm{~F}$ & $60-70$ & $<3$ & 1 & $3-4$ & DM \\
\hline 3 & $\mathrm{~F}$ & $50-60$ & $<3$ & 5 & $3-4$ & DM \\
\hline & $\mathrm{F}$ & $50-60$ & $<3$ & 10 & $1-2$ & HTN \\
\hline 5 & $\mathrm{M}$ & $60-70$ & $<3$ & 16 & $1-2$ & DM \\
\hline 6 & $\mathrm{M}$ & $60-70$ & $<3$ & 0.5 & $<1$ & HTN \\
\hline 7 & $\mathrm{M}$ & $50-60$ & $<3$ & 6 & $3-4$ & HTN \\
\hline 8 & $\mathrm{M}$ & $50-60$ & $<3$ & 10 & $1-2$ & Both \\
\hline 9 & $\mathrm{~F}$ & $50-60$ & $<3$ & 1 & $1-2$ & Both \\
\hline
\end{tabular}

DM, diabetes mellitus; HTN, hypertension; PHC, primary healthcare centre. 
Table 3 Characteristics of patients enrolled for care of DM or HTN at the PHCs

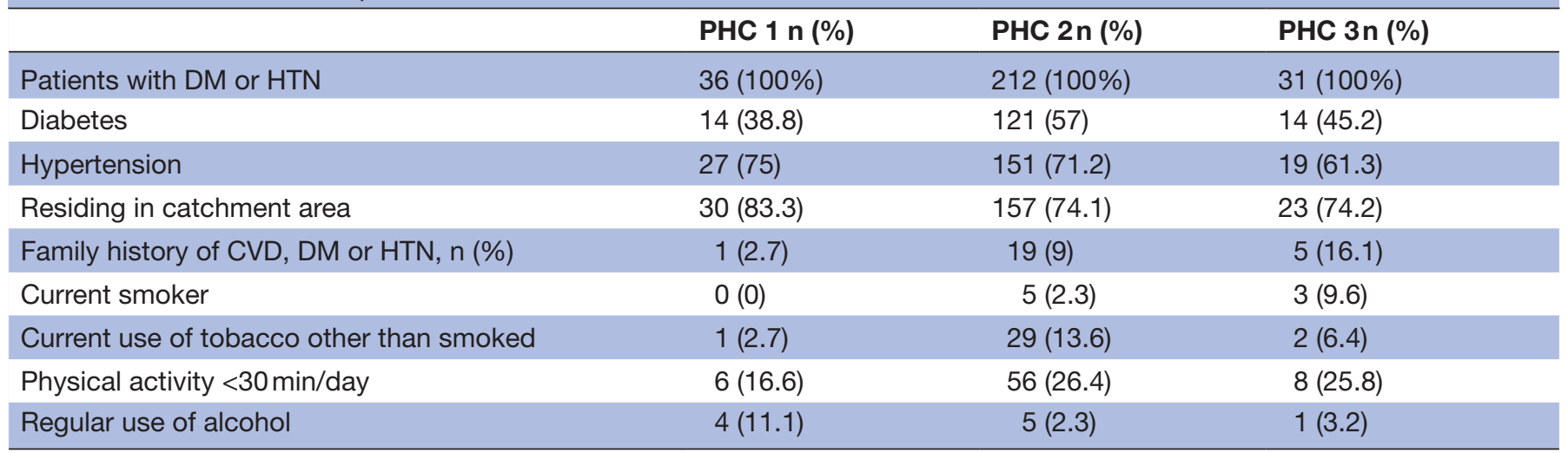

Current smoker-smoked in the past 30 days, regular use of alcohol-consumes alcohol 6 times a week.

CVD, cardiovascular disease; DM, diabetes mellitus; HTN, hypertension; PHC, primary healthcare centre.

and 3, respectively. More patients sought care for hypertension compared with diabetes, and most patients came from the catchment area of the three PHCs. (Table 3) presents the characteristics of the NCD patients attended to in the three PHCs.

The risks assessed at each $\mathrm{PHC}$ visit were related to lifestyle factors known to increase risk for diabetes or hypertension. These factors were tobacco use (smoked or consumed in other forms), physical activity of $<30 \mathrm{~min} /$ day and excessive use of alcohol (standard definitions of the national programme were used). These are selfreported behaviours by the patient with no objective verification, which may explain the wide variations among PHCs.

\section{What changed?}

The workflow with essential tasks was implemented at all three PHCs for the first few months (up to 4 months) of the intervention period with some variation. Patients diagnosed with diabetes or hypertension were directed to have their blood pressure and/or blood glucose measured on arrival at the PHC. During blood pressure measurement, the nurse assessed risk, gave lifestyle advice and directed the patient to consult the doctor. The doctor had information regarding these tasks to make guideline-based

\section{Percentage of persons for follow up at each PHC}

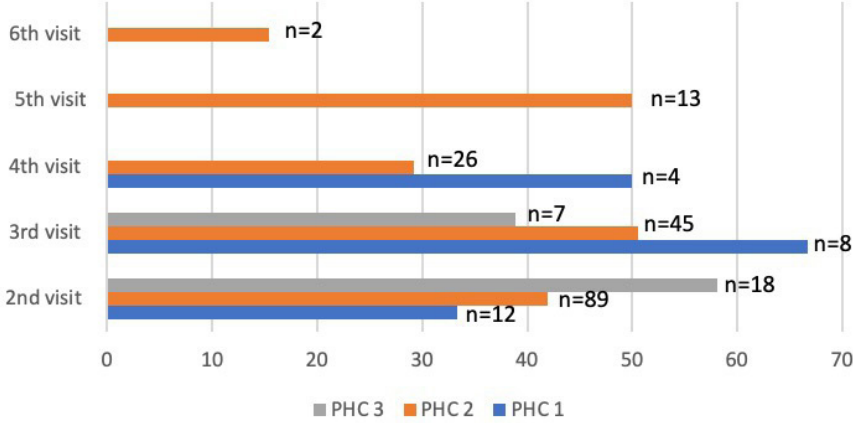

Figure 6 Percentage of patients who came for a scheduled follow-up visit to the primary healthcare centre $(\mathrm{PHC})$. treatment decisions. Patient information was recorded manually in the health record.

During the first month at each of the PHCs, there were challenges in initiating these changes. A few patients found it difficult to follow the new directions and preferred to consult with the doctor first as they had been doing previously. Also, counselling was a time-consuming process that led to delays in the nurses' routine work and longer queues at the nurses' station for patients other than those with diabetes and hypertension.

We observed in the first 2 months that at each PHC, although nurses had volunteered to counsel patients, they were unable to manage the time to complete this task along with their usual work. It was interesting to observe the redistribution of this task that occurred at all three PHCs spontaneously. At PHCs 1 and 2, the lab technicians offered to do this task during measurement of the blood glucose, expressing that they had relatively more time than the nurses; at PHC 3, the doctor completed this task. At PHC 3, the doctor chose to complete risk assessment and enter the information in the clinical records. As a result, at PHC 3, no tasks were shared other than measurement of blood glucose and blood pressure, by the lab technician and nurse, respectively.

The extent to which the essential tasks in the workflow were implemented at each of the PHCs varied considerably. At PHC 3, all tasks were completed for almost all persons enrolled at the PHC (see figure 7). However, these tasks were not performed after July (4 months after start date). At PHC 1, the tasks were not implemented after June (3 months after start date), and at PHC 2, the workflow and the essential tasks continued to the end of the intervention period. However, these were not performed consistently for all patients. Risk assessment and blood pressure and blood glucose measurements were done for most patients $(>60 \%)$. Foot examination was irregularly done and for a few patients only, with no clear pattern emerging. Counselling was done for up to $72 \%$ of patients in June but declined to a low of only $32 \%$ in subsequent months. 

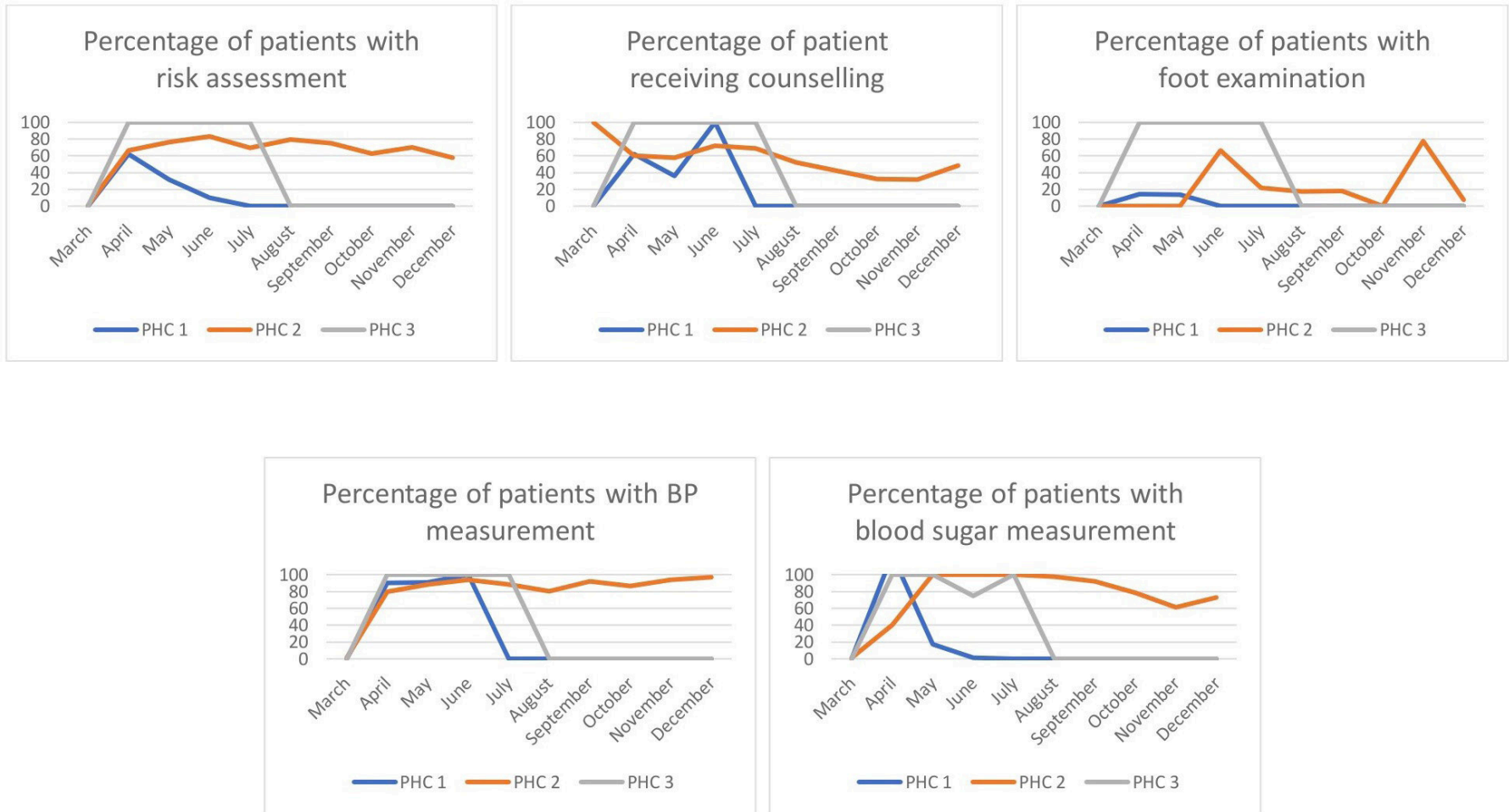

Figure 7 Proportion of patients at the three primary healthcare centres (PHCs) with essential tasks performed during the months (March-December 2018) of implementation. BP, blood pressure.

These changes were perceived positively by most patients who came for more than three follow-up visits. Patients were aware of the changes made in the workflow, and the clinical record was the most tangible difference that they could identify when comparing processes before and after the intervention period. Most patients felt that the workflow was a positive change and made consultation at the clinic more efficient, as expressed in the quotes below:

First at the entrance, we show this book (note book that is retained by patients), they will give us the card (facility retained patient information) and we get blood pressure and blood tests done, then we are in the queue to see the doctor. One by one, [we] go with the card, it becomes easy. That's what we have been discussing, now it's better.-P9

BP will be checked. Advice is given. Tablets are given.-Man, 50-60 years

After they had started giving cards (facility retained patient information), they have said to come once a month. When I come, I will collect the card, [and] once $\mathrm{BP}$ and blood sugars are documented, I show it to the doctor.-Woman, 60-70 years

\section{What did not change?}

The ASHA workers were to make home visits and support patients in the home. These workers also were to remind patients about follow-up visits, but this change was not implemented at any of the three PHCs. We conducted one session at each of the PHCs with the ASHA workers.
At these sessions, the medical officer and the research team discussed with ASHAs the interventions and rationale for the need for follow-up at home. Although ASHAs responded positively to the suggestion of making home visits, saying that they knew most patients in their communities, they were unable to systematically follow-up patients and remind them.

Follow-up by patients was poor and did not improve despite the interventions. There was a steady increase in the number of new patients enrolled at the PHCs in the first 6 months. However, only $60 \%$ of patients enrolled (PHC 3) returned for a second (follow-up) visit.

At PHC 1 and PHC 3, the changes initially made did not continue beyond June and August, respectively. At PHC 3 , the workflow reverted to the same as before the interventions. Tasks were also not shared at PHC 3 and were completed by the doctor. There was no recording of clinical information at either PHC after the first few months.

\section{Why did some of the intended changes occur variably and some not at all?}

There was a relative failure of implementation beyond a few months at both PHC 1 and PHC 3. At PHC 1, the pharmacist and nurse were transferred to another PHC to address a shortage. The lab technician and the doctor continued the tasks, but a few months later, the doctor also left the team because of a promotion to the district office. Staff transfers are not uncommon at PHCs, but three transfers within 2 months was unusual and led to a collapse of the team. We had discussed this possibility with the district health office before the start of 
the interventions but were unable to ensure stability of the team at PHC 1. The workflow became redundant as members of the team were not present and no replacements were made at the PHC in the subsequent months.

At PHC 3, the workflow reverted to as before the interventions, and the doctor preferred to complete tasks without sharing them among team members. In the later months of the intervention, we observed that the doctor counselled patients and used guidelines for treatment but stopped recording this information because it was time consuming. We have previously reported that the local context at PHC 3 was characterised by a strong hierarchy and poor cohesion of the team (accepted for publication). These contextual factors may have contributed to this change. Patient preference for seeing only the doctor may also have had a role. This preference emerged as a theme from the qualitative analysis of the interviews with the patients. Most patients expressed that they attended at the PHC to consult the medical doctor and preferred to ask the doctor for advice regarding their condition.

I come to see the doctor, I will ask the doctor only...Woman, 50-60 years

Only the doctor is able to take care of us well (responding to questions regarding experience of teambased care).-Woman, 60-70 years

Now the doctor should only give advice.-Woman, 50-60 years

ASHAs' role in following up at the home and reminding patients to attend their next visit did not transpire at any of the PHCs. This was hypothesised in the ToC as a pathway to increase follow-up, and the lack of regular follow-up by most patients strengthens this hypothesis. ASHAs lacked opportunities to retrieve information from the clinical records to identify persons who were due for a follow-up visit. There were monthly meetings held at the PHC to discuss national programme implementation. Doctors could have used this forum to discuss follow-up needs of patients with diabetes and hypertension, but this did not happen. Furthermore, ASHAs did not have a monitoring plan to enable supervision of home visits made for diabetes and hypertension. We also observed that ASHAs were fully occupied with maternal and child health-related activities and reports, leaving little time for this other task.

\section{Why did PHC 2 change and what effect did it have on outcomes of care?}

An important resource at PHC 2 was the additional staff member available. This staff person became a coordinator or navigator to guide patients through the workflow. The coordinator was the focal point of contact for the patient. The coordinator also stored and retrieved the clinical records, ensuring completeness. In the local context of PHC 2, we have previously reported that the team was cohesive and that the doctor encouraged sharing of tasks (accepted for publication). These factors
Mean fasting blood glucose levels by personmonths of follow-up

400

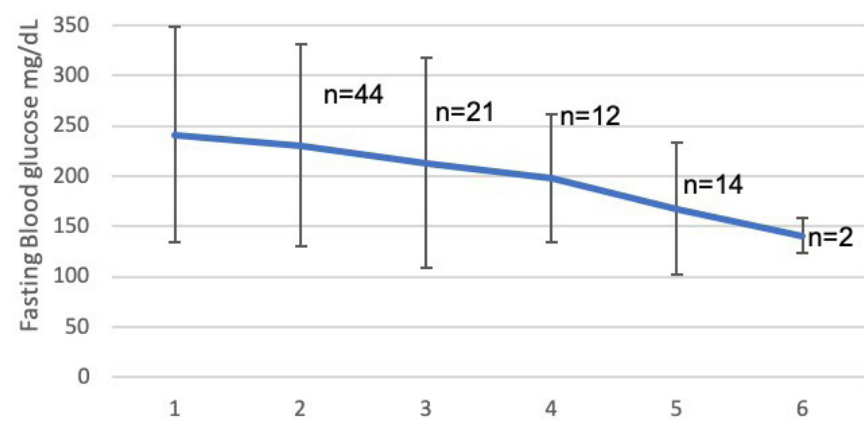

Figure 8 Average fasting blood glucose levels by personmonths exposure to interventions (error bars: SD).

may have contributed to the relative success of the interventions at PHC 2.

Most patients expressed that they felt they had achieved better control of their conditions. This positive feedback may also have contributed to the changes that we observed at PHC 2, as illustrated in the following quotes and supported by the outcomes assessed:

My sugar has decreased. Earlier it was 350.-Woman, $60-70$ years

Now [my] blood pressure is good. It is 130. I have been coming for one year. I feel my health is improved now.-Woman, 50-60 years

$\mathrm{BP}$ has come down. If it is more, then they advise to take less salt and spice.-Man, $60-70$ years

\section{Outcomes at PHC 2}

The clinical outcomes were assessed only at PHC 2, where implementation was continued for the entire 9-month period and patients were able to complete $5(\mathrm{n}=13)$ and $6(\mathrm{n}=2)$ follow-up visits (figure 6$)$. The main outcome of interest was glycaemic and blood pressure control. As hypothesised in the ToC, we found a decline in both the average fasting blood glucose and systolic blood pressure with an increase in the person-months of follow-up (figures 8 and 9).

\section{DISCUSSION}

In this study at three PHCs in rural South India, we co-designed interventions with implementers to strengthen primary care for diabetes and hypertension. The interventions included an optimisation of the workflow to incorporate essential tasks into the care of diabetes and hypertension, distribution of tasks among members of primary care teams, introduction of clinical records to be retained at the health facility and involvement of ASHAs for follow-up of patients. All three PHCs implemented the interventions for the first 4 months, however at two of the three PHCs, implementation did not continue. This was most likely due to the transfer of team members at 
one of the PHCs and team dynamics that did not support task sharing at the other. Patients who came for regular follow-up visits (more than three visits during 9 months of implementation) to the PHC showed a reduction in blood pressure and blood glucose levels.

This study used participatory approaches, involving the implementers in the design, development and implementation of the interventions. These approaches are known to result in changes in practice and also foster implementers' ownership of the interventions. ${ }^{31}$ In our study, we found that this approach did lead to some ownership and involvement of the team in implementing the interventions, as evidenced by a redistribution of tasks, different from the initial plan, that occurred in the second month of implementation. However, this approach also meant that when the staff at PHC 1, for example, chose not to continue implementation of the interventions after 4 months, we as researchers could not enforce adherence to the programme, as may have been done in traditional standardised approaches and research designs. Research in the real-world setting required us to acknowledge and embrace the lack of 'control' in the setting.

The staff transfers that occurred at PHC 1 were also beyond the control of the local team at the PHC and beyond our control as researchers. Despite having discussed it with the district office during the selection of the PHCs, we could not prevent this from occurring. We found that the lack of continuity in staff at the PHC limited continuity of care, which is believed to be a fundamental attribute of quality of care for chronic conditions. ${ }^{4} 10$ Haggerty et al described three types of continuity in care that are relevant across different disciplines: relational, informational and management. ${ }^{32}$ 'Relational continuity' references the continuous relationship patients develop with their primary care provider, and 'informational continuity' refers to the availability of clinical records and management continuity in the treatment of the condition. Relational continuity is valued in primary care and conveyed through the presence of a consistent core of staff. This consistency provides patients with a sense of

\section{Systolic BP by person-months of follow-up}

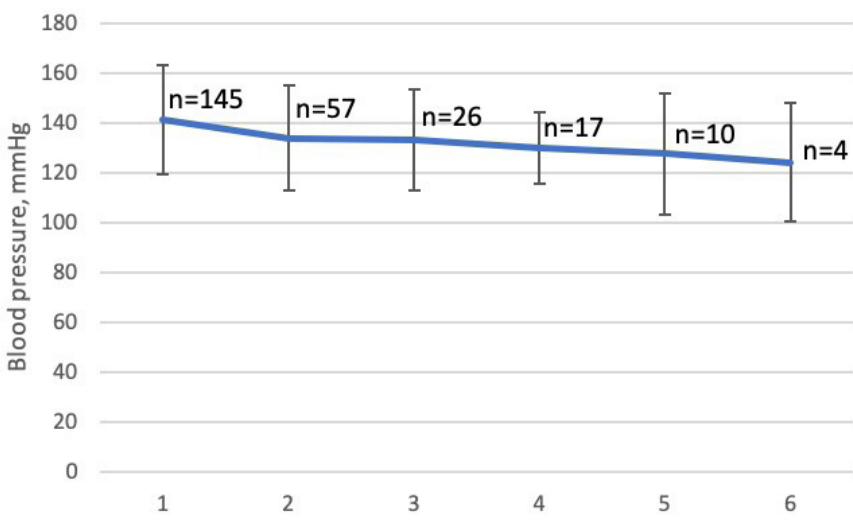

Figure 9 Average systolic blood pressure (BP) by personmonths of exposure to the interventions (error bars: SD). predictability and coherence in seeking care for their chronic condition. ${ }^{32}$ Frequent staff transfers and instability within teams in primary care in the Indian context are a threat to achieving relational continuity with patients. Sheikh et al also point to the negative impact such transfers have on the trust people place in government institutions. ${ }^{33}$ Ensuring that at least a minimum core staff such as the nurse or doctor will remain in continuous service at a PHC for a specified length of time may be a way to overcome this challenge. Less specialised staff are more likely to be available for longer periods, and PHC models that are nurse-led or coordinator-led thus should be explored in the Indian context. ASHA could potentially also play a role in maintaining continuity. However, we did not succeed in involving ASHAs in patient follow-up, possibly because of a lack of specific incentives to do so. Studies have indicated that a too-heavy workload with little compensation results in demotivation of ASHAs. ${ }^{34}$ The state of Karnataka recently experienced a protest by $>15000$ ASHA workers demanding an increase in the honorarium they receive from the government. ${ }^{35}$

We found that the presence of a coordinator at PHC 2 facilitated the implementation of interventions. The coordinator became the contact person for patients, directing them to complete the various tasks at the PHC, maintaining the clinical records and retrieveing them at each follow-up visit by the patient. In our study, the role of a coordinator was not part of the interventions but evolved as a local solution to the need for someone to manage the clinical records. The role of navigators in helping patients negotiate the healthcare delivery system, linking the health services with social services has been described in the literature regarding primary care services. ${ }^{36}$ However, there is need for a role that can ensure continuity, help patients navigate care processes and coordinate care. This role is underexplored in the Indian context and could potentially improve the quality of care for persons with a chronic condition. This possibility is especially relevant because the recent National Medical Bill 2019 proposes the role of a mid-level healthcare provider. This function has not been clearly articulated but is envisaged as supporting primary healthcare delivered at health and wellness centres. A role for care coordination or navigation could be a possibility for this new team member in primary care.

The National Programme for Prevention and Control of Cancer, Diabetes, Cardiovascular diseases and Stroke in India prescribes the package of services at a PHC. The services for diabetes and hypertension include counselling by the nurse, population-based/opportunistic screening for diabetes and hypertension by the auxiliary nurse midwife, clinical diagnosis and treatment, referral of complicated cases and follow-up of patients under treatment by the doctor. ${ }^{37}$ However, insufficient guidance is provided for how these services will be integrated into the functions of a PHC. In our study, at all three sites, the nurse was unable to counsel patients. Based on our experience, we recommend that team-based care be included in 
the guidelines and that role clarity be provided, keeping in mind all activities at a PHC and the possibility of new roles, such as care coordination, to strengthen care for chronic conditions.

\section{Reflections on the ToC approach}

The ToC approach enabled us to clearly articulate the outcomes aimed for and clarify the resources deemed necessary to create change, as well as to hypothesise how the change would happen. We used the ToC to design the interventions and to identify the evaluation methods and indicators for both processes and outcomes. An integrative framework combining process and outcome measurement is the biggest strength of this approach. ${ }^{38}$ However, we also found that the ToC was too linear in its articulation of change but that change did not occur in such a manner. The challenges and local solutions that developed in the course of the implementation were difficult to place in the theory during implementation. Furthermore, we found it difficult to represent the flexibility of the participatory methods we chose and the fine-tuning of the interventions that occurred. The assumptions we made may explain the changes that occurred in the first few months at the PHCs. The transfer of staff (PHC 1) and the doctor's preference for completing tasks (PHC 3 ) were unexpected occurrences that were not accounted for in the ToC. Action research designs may be an alternative enabling greater represenataion of the necessary flexibility to adapt interventions in the course of the planning and action cycles, central to the methodology.

Change, especially in work patterns, is difficult for primary care teams to achieve alongside the usual activities of a busy primary care facility. Our role as the research team was crucial in organising meetings and facilitating the changes that the teams could implement. Based on our experience, we propose that facilitation of quality improvement in primary care teams in the Indian context is required. Staff in primary care would benefit from the support that an external team can provide. This support could be in the form of assistance in collating monitoring data and feedback to the team or availability for troubleshooting in case of difficulties. External facilitation for quality is reported in the primary care literature as a successful strategy to bring about change. ${ }^{16}$ Quality improvement research with external facilitation to experiment and innovate in primary care settings has the potential to transform primary care in India.

\section{Limitations}

A major limitation of our study is the lack of involvement of patients as important stakeholders in the development and design of the interventions. We were unable to bring patients and community representatives to the discussions with the PHC staff regarding the design of the interventions. The staff at PHC were not keen to involve patients or a community representative. This reluctance may have been the result of local traditions that do not allow for involvement of patients in discussions regarding treatments and organisation of care.

Another limitation of the study was the short period of 9 months allotted to implement the interventions and assess the effects. Change is difficult to establish and sometimes may result after years of persistence. It is possible that follow-up for another year might have yielded different results with regard to the implemented processes. We also were limited by a reliance on clinical records as the only source of data for clinical outcomes. However, we had no other source of data at the PHC that we could use to verify the data in the clinical record.

The findings of this study are limited to three rural PHCs in Karnataka. The local context plays a huge role in implementation, so the findings are not generalisable even to different PHC settings in India. However, the use of the ToC and the analytic lens of what changed and why may offer insights for other, similar settings. We also acknowledge the narrow focus of the interventions on service delivery for persons with diabetes and hypertension. Prevention and promotion is an essential function of primary care but were not studied.

\section{CONCLUSION}

In this implementation research study, we co-designed an intervention package and facilitated its implementation at three publicly funded primary care centres. We found a variable response at each of the three PHCs. Implementation was limited at one because of transfer of team members and at another because of the doctor's preference for completing tasks without involving other team members. Only one of the PHCs could change and adopt the interventions throughout the 9 months of implementation, to varying extents. We found that transfer of staff is a threat to providing quality care for chronic conditions. Stable core staff are needed to ensure relational continuity at primary care facilities. We also found that the presence of a coordinator to assist patients in navigating the processes of care facilitated the care delivered at primary care centres. We recommend that this role be considered for the mid-level health provider proposed in the recent healthcare reforms in India. Our role as researchers in co-designing and facilitating the interventions was crucial in bringing about change at the health facilities, and we advocate for external support to primary care teams to innovate and redesign care. These findings draw attention to teams in primary care and their composition, roles and leadership. Strengthening the team will contribute to delivering care for chronic conditions, especially in resource-constrained settings such as India.

\section{Author affiliations}

${ }^{1}$ Health Services, Institute of Public Health Bengaluru, Bangalore, Karnataka, India ${ }^{2}$ CAPHRI Care and Public Health Research Institute, Faculty of Health and Medicine and Life Sciences, Maastricht University, Maastricht, Limburg, The Netherlands ${ }^{3}$ Health Equity Research, Institute of Public Health Bengaluru, Bangalore, Karnataka, India

${ }^{4}$ Primary Care, Health Systems Transformation Platform, New Delhi, India 
${ }^{5}$ Department of Public Health, Institute of Tropical Medicine, Antwerp, Belgium

Acknowledgements The authors would like to acknowledge the assistance of Prashanthi Kamath in data collection and field notes that contributed to the data analysis. The authors would like to acknowledge the participation of the teams at the three facilities, especially the medical officers that shaped and co-designed the interventions. The authors would also like to acknowledge the patients at the facilities that allowed to make and accept the changes made at the facilities.

Contributors DL, ND and BC designed and planned the study. DL, ND, BC, NE and $\mathrm{KH}$ participated in analysis of the data. $\mathrm{DL}$ and $\mathrm{BC}$ conceived the paper and this was substantially shaped by KH, PNS and NE. All authors contributed to the manuscript and approve of the final submission.

Funding The primary author and the research was supported by a PhD scholarship from the Institute of Tropical Medicine, Antwerp, Belgium. PNS time contributions were supported by a DBT/Wellcome Trust India Alliance fellowship (number IA/ $\mathrm{CPHI} / 16 / 1 / 502648$ ) awarded to him.

Competing interests None declared.

Patient and public involvement Patients and/or the public were not involved in the design, or conduct, or reporting, or dissemination plans of this research.

Patient consent for publication Not required.

Ethics approval The study received ethical approval from the ethics committees at the Institute of Tropical Medicine, Antwerp, the University of Antwerp, Belgium and the Institute of Public Health in Bengaluru, India.

Provenance and peer review Not commissioned; externally peer reviewed.

Data availability statement Data are available on reasonable request. The data are available with the corresponding author and will be shared on reasonable request. We have not stored in a publicly accessible data base as the qualitative information may allow identification of location and site.

Open access This is an open access article distributed in accordance with the Creative Commons Attribution Non Commercial (CC BY-NC 4.0) license, which permits others to distribute, remix, adapt, build upon this work non-commercially, and license their derivative works on different terms, provided the original work is properly cited, appropriate credit is given, any changes made indicated, and the use is non-commercial. See: http://creativecommons.org/licenses/by-nc/4.0/.

\section{ORCID iD}

Dorothy Lall http://orcid.org/0000-0002-2776-2182

\section{REFERENCES}

1 GBD 2017 Causes of Death Collaborators. Global, regional, and national age-sex-specific mortality for 282 causes of death in 195 countries and territories, 1980-2017: a systematic analysis for the global burden of disease study 2017. Lancet 2018;392:1736-88.

2 International Institute for population sciences (IIPS) and ICF. National family health survey (NFHS-4). Mumbai.

3 European Observatory on Health Systems and Policies Series. Caring for people with chronic conditions : a health system perspective. England: Mc Graw Hill, 2008. http://www.euro.who. int/_data/assets/pdf_file/0006/96468/E91878.pdf

4 Allotey P, Reidpath DD, Yasin S, et al. Rethinking health-care systems: a focus on chronicity. Lancet 2011;377:450-1.

5 Sinha R, Pati S. Addressing the escalating burden of chronic diseases in India: need for strengthening primary care. J Family Med Prim Care 2017;6:701.

6 Varghese C, Nongkynrih B, Onakpoya I, et al. Better health and wellbeing for billion more people: integrating non-communicable diseases in primary care. BMJ 2019;364:I327-3.

7 World Health Organization. Primary health care now more than ever. Geneva: WHO, 2008.

8 World Health Organization. Innovative care for chronic conditions. Geneva: World Heal Organ, 2001.

9 Lall D, Engel N, Devadasan N, et al. Models of care for chronic conditions in low/middle-income countries: a 'best fit' framework synthesis. BMJ Glob Health 2018;3:e001077-12.

10 Kruk ME, Nigenda G, Knaul FM. Redesigning primary care to tackle the global epidemic of noncommunicable disease. Am J Public Health 2015;105:431-7.

11 Bhojani U, Devedasan N, Mishra A, et al. Health system challenges in organizing quality diabetes care for urban poor in South India. PLoS One 2014;9:e106522.
12 Elias MA, Pati MK, Aivalli P, et al. Preparedness for delivering noncommunicable disease services in primary care: access to medicines for diabetes and hypertension in a district in South India. BMJ Glob Health 2017;2:e000519.

13 Pakhare A, Kumar S, Goyal S, et al. Assessment of primary care facilities for cardiovascular disease preparedness in Madhya Pradesh, India. BMC Health Serv Res 2015;15:408.

14 Grover A, Joshi A. An overview of chronic disease models: a systematic literature review. Glob J Health Sci 2014;7:210-27.

15 Coleman K, Austin BT, Brach C, et al. Evidence on the chronic care model in the new millennium. Health Aff 2009;28:75-85.

16 Lau R, Stevenson F, Ong BN, et al. Achieving change in primary care-causes of the evidence to practice gap: systematic reviews of reviews. Implement Sci 2016;11.

17 Craig P, Dieppe P, Macintyre S, et al. Developing and evaluating complex interventions: Following considerable dveelopment in the field since 2006, MRC and NIHR have jointly commisioned and update of this guidance to be published in 2019. Med Res Counc Published Online First, 2019. Available: https://mrc.ukri.org/ documents/pdf/complex-interventions-guidance/

18 Lall D, Engel N, Devadasan N, et al. Challenges in primary care for diabetes and hypertension: an observational study of the Kolar district in rural India. BMC Health Serv Res 2019;19:44.

19 Lall D, Engel N, Narayanan Devadasan KH, et al. Team-Based primary health care for non-communicable diseases: complexities in South India. Press, 2020.

20 Breuer E, Lee L, De Silva M, et al. Using theory of change to design and evaluate public health interventions: a systematic review. Implement Sci 2016;11.

21 Ministry of Health \& Family Welfare- Government of India. Indian Public Health Standards (IPHS) Guidelines for Primary Health Centres. Indian Public Heal Stand. 1-100, 2012. Available: http:// nhm.gov.in/images/pdf/guidelines/iphs/iphs-revised-guidlines-2012/ primay-health-centres.pdf

22 Services DG of $\mathrm{H}$, India M of H \& FWG of. Indian public health standards (Iphs) guidelines for primary health centres revised, 2012. Available: http://health.bih.nic.in/Docs/Guidelines/Guidelines-PHC2012.pdf

23 WHO. Package of essential noncommunicable (Pen) disease interventions for primary health care in low-resource settings. France: World Health Organisation Press, 2010.

24 Ministry of Health \& Family Welfare, Government of India. Operational guidelines, 2010. Available: http://www.who.int/lep/resources/ SEAGLP20062.pdf

25 National Centre for Disease Control, MOHFW, GOI. National programme for prevention and control of cancer, diabetes, cardiovascular diseases and stroke training module for medical officers for prevention, control and population level screening of hypertension. Diabetes and Common Cancer, 2017. Available: http:// www.nicd.nic.in/writereaddata/mainlinkFile/File637.pdf

26 Weiss $\mathrm{CH}$. Nothing as practical as good theory: exploring TheoryBased evaluation for comprehensive community initiatives for children and families. New Approaches to Eval Community Initiat Concepts, Methods, Context 1995:65-92.

27 De Silva MJ, Breuer E, Lee L, et al. Theory of change: a theory-driven approach to enhance the medical Research Council's framework for complex interventions. Trials 2014;15:267.

28 Anderson AA. The community builder's approach to theory of change. Aspen Inst 2005;37.

29 Damschroder LJ, Aron DC, Keith RE, et al. Fostering implementation of health services research findings into practice: a consolidated framework for advancing implementation science. Implement Sci 2009;4:40-55.

30 Kaplan HC, Provost LP, Froehle CM, et al. The model for understanding success in quality (MUSIQ): building a theory of context in healthcare quality improvement. BMJ Qual Saf 2012;21:13-20.

31 Peters DH, Tran NT, Adam T. Implementation research in health: a practical guide, 2013. Available: http://who.int/alliance-hpsr/ alliancehpsr irpguide.pdf

32 Haggerty JL, Reid RJ, Freeman GK, et al. Continuity of care: a multidisciplinary review. BMJ 2003;327:1219-21.

33 Sheikh K, Freedman L, Ghaffar A, et al. Posting and transfer: key to fostering trust in government health services. Hum Resour Health 2015;13:80-3.

34 Saprii L, Richards E, Kokho P, et al. Community health workers in rural India: analysing the opportunities and challenges accredited social health activists (ASHAs) face in realising their multiple roles. Hum Resour Health 2015;13:95.

35 The Hindu. Karnataka: ASHA workers launch massive strike. Newspaper, 2020. Available: https://www.thehindu.com/news/ 
national/karnataka/asha-workers-launch-massive-strike/ article30468080.ece [Accessed 21 Feb 2020].

36 Carter N, Valaitis RK, Lam A, et al. Navigation delivery models and roles of navigators in primary care: a scoping literature review. BMC Health Serv Res 2018;18:1-13.
37 Ministry of Health \& Family Welfare. Operational Guidelines - Prevention, Screening and Control of Non Communicable Diseases, 2016

38 De SM, Lee L. Using theory of change in the development, implementation and evaluation of complex health interventions. A practical guide. London Sch Hyg Trop Med Cent Glob Ment Heal 2014:1-14. 